\title{
Una propuesta de análisis de los estados latinoamericanos desde las políticas públicas
}

\author{
Leonel Flores Vega \\ El Colegio de México A.C., Ciudad de México, México. \\ Email: lfloresv@colmex.mx
}

\begin{abstract}
Resumen: Los temas de investigación política en la década de los sesenta se centraron en la revolución, en los ochenta lo fue la democracia, en los noventa la reforma del Estado, sin embargo, el tema subyacente a estos fue y sigue siendo el tipo de políticas que diseñan e implementan los Estados latinoamericanos. El desafío es cómo hacer operativo el análisis de los países y al mismo tiempo cómo hacerlos comparables con otros. Por ello, se propone analizar la acción pública desde las políticas públicas definidas, diseñadas e implementadas por estructuras institucionales para atender asuntos públicos. Con ello se propone analizar la naturaleza y transformación de los países de América Latina.
\end{abstract}

Palabras clave: América latina, estado, políticas públicas, acción pública

\section{A proposal for analysis of Latin American States from public policies}

\begin{abstract}
Political research topics in the 1960s focused on the revolution; in the 1980s, the discussion was about democracy; in the 1990s state reform; however, the underlying theme of these was and remains the type of policies designed and implemented by Latin American states. The challenge is how to make operational the analysis of countries and how to make them comparable to others. Therefore, it is proposed to analyze public action from public policies defined, designed and implemented by institutional structures to address public issues. It proposes to analyze the nature and transformation of the countries of Latin America.
\end{abstract}

Keywords: Latin America, State, public policies, public action

\section{Uma proposta de análise de estados latino-americanos a partir das políticas públicas}

Resumo: Os temas da pesquisa política nos anos 60 focados na revolução, na década de oitenta centraram-se no tema da democracia e na década de noventa, a reforma do Estado. No entanto, o tema subjacente era e continua sendo o tipo de políticas que desenham e implementam os Estados latino-americanos. O desafio é como fazer operativa a análise dos países e simultaneamente como torná-los comparáveis uns aos outros. Portanto, propõe-se analisar a ação pública a partir das políticas públicas definidas, desenhadas e implementadas por estruturas institucionais para abordar questões públicas. Propõe analisar a natureza e a transformação dos países da América Latina. 
Palavras-chave: América Latina, estado, políticas públicas, ação pública

\section{Introducción: de la revolución al análisis de políticas públicas}

El grupo de países que integran América Latina no son homogéneos, sin embargo, en las ciencias sociales se ha elaborado una "construcción intelectual”, como lo plantean Yocelevzky y Cuellar (2011), de un grupo de estados nacionales. La historia contemporanea de América Latina tiene una coyuntura definida por la crisis de finales de los años veinte y el término de la segunda guerra mundial. Según Halperin Donghi (2013) los cimientos del orden económico latinoamericano se volvieron endebles. Esto dio pie al diseño de modelos y políticas para reimpulsar el crecimiento en Estados con instituciones débiles y profundas desigualdades (Filgueira, 2009). Aundado de una construcción teórica con diversas explicaciones que van desde el desarrollismo, pasando por la teoría de la modernización y la teoría de la dependencia en sus diversas etapas.

Las transformaciones políticas que tuvieron este grupo de países en la segunda mitad del siglo XX influyeron, entre otros muchos temas, en las prioridades de investigación política. Si bien, Lechner (1988) plantea que en la década de los sesenta el tema central era la revolución y en los ochenta fue la democracia, ambos tópicos se presentan como condición necesaria para el “desarrollo del subdesarrollo”, al mismo tiempo, como un campo de conocimiento para el debate intelectual. Siguiendo esta línea, la década de los noventa fue un periodo de democratización con transiciones de dictaduras a gobiernos representativos con elecciones. En los países con elecciones periódicas, hubo actores hegemónicos con poca legitimidad. También se ampliaron las garantías de la oposición, como el caso mexicano, esto también fue conocido como la primera oleada de la reforma del Estado ${ }^{1}$ (Andara M., 2007).

Los temas de la revolución y la democracia han sido fuertemente modificados por la historia reciente, pero en lo grueso siguen siendo los mismos, aunque ahora discutiendo en un ámbito de transformación polifacética del Estado (Bolívar Espinoza \& Flores Vega, 2014). Si anteriormente, la acción pública del Estado era el producto de los gobernantes, donde el eje analítico era quién y cómo gobierna, en la década de los noventa, el Estado inició a estudiarse en tanto su acción reflejada por medio de las políticas públicas, es decir, cómo y quién elabora e implementa una política pública (Meny \& Thoenig, 1992; Roth Deubel, 2006). Con lo anterior, aumentaron los estudios preocupados por la acción concreta del Estado, teniendo presente a las políticas, los programas de gobierno, las instituciones y los actores, en oposición a los análisis de partidos, parlamentarismo, representación política, etc. 
El análisis de políticas públicas identifica actores concretos que intervienen en la definición de una problemática, en el diseño de una acción orientada a resolver un problema, así como de su implementación y evaluación de dicha acción para conocer su eficacia. La finalidad es poner en la mesa de debate las posiciones, intereses y objetivos de cada uno de los actores en el ciclo de las políticas, sean gubernamentales o sociales. Algunos autores reconocen estos planteamientos como la ciencia del Estado en acción por la ejecución de metodologías para el análisis de la actividad concreta de las autoridades públicas (Jobert \& Muller, 1987; Jobert, 1994).

El análisis de las políticas públicas aporta elementos teóricos y empíricos para comprender dificultades de modelos de Estado como el del bienestar, el corporativismo, el neoliberalismo, por mencionar algunos. El tema transversal es cómo comprender la naturaleza y evolución de los Estados y de esta manera explicar el rumbo de cada país. Según Meny y Thoening (1992) durante los años sesenta el tema fundamental fue el análisis y comparación de los Estados providencia, en los ochenta los fue la constitución de agendas gubernamentales y la organización de los actores. En los noventa, señala Roth Deubel (2006) la ola neoliberal impulsó el debate de una nueva gestión pública y se incrementó el interés por la evaluación de las políticas.

La propuesta que ponemos sobre la mesa es analizar las políticas públicas para caracterizar y definir el tipo de Estado. Por ejemplo, los países que en América Latina han experimentado, desde la década de los sesenta, además de los golpes de Estado, revoluciones y alternancias sorpresivas, se preocupó por la ineficacia y racionalización de las burocracias. Los regímenes políticos en América Latina cambiaron y hubo efectos en las condiciones de funcionamiento del Estado en el sistema económico y político. Aparecieron diversas políticas e instituciones públicas, y una participación social creciente aunque, no han faltado inéditas formas de delincuencia y violencia trasnacionales que dejan a los países latinoamericanos al borde de la ingobernabilidad (O’Donnell, 2004). El papel del Estado en los países latinoamericanos es innegable, ya sea para definir su capacidad de organización, para determinar el cambio de sus funciones centrales o para aplicar políticas públicas, lo cierto es que no puede actuar o cambiar sin un sistema institucional (Evans, 2001). El estudio de las políticas públicas, como lo señala Oszlak y O’Donnell (1976) es una forma de contribuir al conocimiento de los estados latinoamericanos. Es decir, las políticas son un instrumento heurístico para comprender la acción y el funcionamiento de los Estados (Bolívar Espinoza \& Flores Vega, 2014).

En este trabajo se compone de dos apartados: en el primero se discute la agonía y reaparición del Estado como concepto y campo analítico para entender las acciones públicas, destacando el papel de los enfoques pluralistas y estatistas, ante ello, se presenta una propuesta operativa de análisis del Estado para hacer frente al haló metafísico con el que históricamente se creó. En la segunda parte, se presenta el análisis de las políticas públicas como instrumento heurístico para comprender el funcionamiento 
de cualquier Estado. La pretensión es superar los análisis centrados en el ciclo de las políticas públicas ${ }^{2}$ o en alguna de sus etapas y la aspiración es caracterizar diacrónica y sincrónicamente a los estados latinoamericanos, lo cual permite estudiar y comparar los países latinoamericanos.

\section{Retorno del Estado}

\section{Agonía del Estado}

La discusión teórica sobre el Estado en la ciencia política, durante gran parte del siglo XX, pareció no estar en el centro de las investigaciones políticas. La fuerza que tuvieron los enfoques pluralistas y estructuralfuncionalistas predominantes en la ciencia política y sociología anglosajona estableció el comportamiento de las élites y su búsqueda por el poder político la forma cotidiana de explicar la política y las actividades gubernamentales centradas en la sociedad. El Estado fue considerado como un concepto viejo con una orientación filosófica y en el mejor de los casos asociado al campo jurídico (Skocpol, 2007). La crítica al Estado se basa en su ambigüedad, tanto en el concepto como en su ámbito efectivo ${ }^{3}$.

Desde estas corrientes de pensamiento político, el Estado fue perdiendo fuerza en el campo de investigación política. Había preocupación por estudiar a grupos de interés y las élites políticas, buscaba a toda costa las razones para acceder al poder político, aun así se negaban a averiguar cuál era el marco de estos actores. Alain Wolfe (1980) lo recupera de manera magistral al señalar lo siguiente:

"Al igual que una novela victoriana que habla de las pasiones sin sexo, los pluralistas intentaron examinar la política sin el Estado (...). David Easton dedicó quinientas páginas al análisis de la 'vida política’ y ni siquiera mencionó una sola vez al Estado-por una cuestión de principios y no de ignorancia. Profundamente apolítico a pesar de su autoproclamado amor al conflicto y a la diversidad, el pluralismo manejó el tema clave de la política moderna pretendiendo que no existía”

El halo metafísico y la existencia paradójica del Estado han llevado a que no sólo se dude de su existencia sino que se le ignore completamente. La literatura especializada anglosajona desde principios del siglo XX habla de todo menos del Estado. En la investigación política surgieron algunos conceptos alternativos como: gobierno o sistema político (Bolívar Espinoza A. , 1997a). El cambio de los conceptos es claro en Robert MacIver quién en 1926 publicó su libro "The modern state”, varios años después el mismo autor publica su libro titulado "The web of government", sustituyendo al Estado por el gobierno (Wolfe, 1980).

Aunado a ello, la preponderancia de los estudios empiristas de dichos enfoques, cuestionaron el carácter intangible el Estado, con lo que se 
acercaba más a la filosofía que a las ciencias sociales. El Estado no fue considerado como un objeto de estudio, con lo cual casi desapareció de la literatura, en su lugar tomó gran importancia “el gobierno” (Bolívar Espinoza A. , 1997a). De acuerdo con Skocpol (2007, p. 170) el gobierno “...era considerado primordialmente como un terreno en el que los grupos de interés de carácter económico o los movimientos sociales normativos contendían o se aliaban entre sí para configurar la adopción de decisiones sobre política pública”.

Los Estados desde la visión de Alfred Stepan (1978) deben ser considerados como algo más que "gobiernos", pues los sistemas administrativos, jurídicos, burocráticos y coercitivos no sólo regulan las relaciones entre la sociedad civil y la autoridad pública, sino que establece cohesión en la sociedad civil (Skocpol, 2007). Además, la organización de la sociedad civil, establece mecanismos de cohesión social que no responden a la racionalidad o maximización de los beneficios, sino a elementos que cohesionan a la sociedad civil. El predominio de los estudios pluralistas, apartaron al Estado como objeto de estudio, incluidos los campos de estudio dedicados al análisis la elaboración de la política pública (Meny \& Thoenig, 1992).

Para entender la debilidad del estatismo como campo de investigación política, simplificadamente podemos señalar que la contradicción es entre el enfoque pluralista y el estatista, aunque hay algunos enfoques más presentes $^{4}$. La perspectiva estatista se preocupa de los procesos políticos de larga envergadura y se centra en los estudios sobre el Estado, especialmente ilustrados por las tradiciones, al menos de Hegel, Marx o Weber (Bolívar Espinoza A. , 1997a).

El enfoque pluralista con la noción de "sistema político" se desarrolla en el espectro de la tesis pluralista en la ciencia política norteamericana de los años cincuenta. Se construye como reacción contra el "estatismo”, a la sobrevaloración de la técnica, a la falta de teoría los estudios políticos. El pluralismo enfatiza el carácter ineludiblemente societal del Estado en la inmersión de las políticas públicas en la complejidad de las relaciones sociales y, sobre todo, en la naturaleza inevitablemente contradictoria de la acción pública, sometida a una multiplicidad de demandas (Muller \& Surel, 1998). Pronto el enfoque angloamericano se hizo hegemónico tanto por la nueva metodología como por el peso de algunos de sus supuestos valóricos democráticos. El concepto de "sistema político” aportó un bagaje conceptual más amplio para interpretar información empírica.

\section{Reaparición del Estado como objeto de estudio}

En las últimas décadas del siglo XX, a pesar de la hegemonía del enfoque pluralista, se incorporaron nuevas perspectivas que retoman al Estado. Por una parte reconocen que el Estado estudiado por los clásicos ha sufrido grandes transformaciones teórica y empíricamente. Por otra par- 
te, los comportamientos de la sociedad no son suficientes para explicarse a ellos mismos y requieren de una dimensión nueva de tratar al Estado.

Varios han sido los intentos por enfrentar y superar, en alguna medida, la contradicción fundamental entre el estatismo y, el pluralismo y sus derivaciones. El debate que produjo la crítica a la perspectiva pluralista del Estado y la reacción dentro de sus propias filas hizo posible un mejor acercamiento al análisis institucional. La renovación de la postura "estatista" reinstaló el estudio institucional mediante el "neo institucionalismo", procedente de las ideas de la escuela neoclásica de la economía política impulsada por Douglas North (1995), donde el papel de las instituciones políticas es establecer las reglas de juego para organizar la sociedad, al mismo tiempo como base para que funcione el sistema social.

Con ello, se iniciaron las bases para reinstalar al Estado en la investigación política. Este papel no respondió a debates abstractos, sino a trabajos con investigación de programas y políticas de los gobiernos para entender su comportamiento. Esta interpretación no está asociada a teorías sistémicas como el funcionalismo o el marxismo (Skocpol, 2007). Las acciones de las autoridades públicas y las demandas sociales son el objeto que ha convocado a este concepto para actualizarlo y darle contenido tangible. La acción del Estado se traduce en la gestión y el ejercicio del poder de manera mezclada (Jobert, 1994). Para ello incluso partimos de una definición tangible del Estado como se explica a continuación.

\section{¿Qué entendemos por Estado?}

La definición del Estado depende del nivel analítico en el que planteemos la discusión, si ponemos la vara muy alta, tendríamos que discutir al menos desde la filosofía, sin embargo, nuestra pretensión es discutir al Estado desde la ciencia y sociología política. El desafío es tener un marco general, retomando los datos empíricos, con ello, las particularidades de los regímenes políticos específicos. Para lograr lo anterior, retomamos la concepción de Estado desde una perspectiva “funcional orgánica” planteada por Gérard Bergeron (1965; 1993), distanciada del enfoque estructural funcional ${ }^{5}$.

El enfoque funcional orgánico de Bergeron (1993) no parte de un concepto a prior del Estado (ni general ni empírico), sino centra su atención en determinar las funciones específicas y detectables. El Estado es visto desde el punto de vista orgánico, sus determinaciones generales y específicas dependen de su actividad concreta. Bergeron (1993) construye la "noción” e “idea” del referente del Estado desde un punto de vista fenomenológico. El funcionamiento del Estado (acciones humanas) antecede a la idea del Estado en funcionamiento (constitucional); se preocupa por determinar las acciones humanas, antes que la reglamentación de dichas acciones. 
La perspectiva del Estado en funcionamiento sugiere fácilmente la relación "función-estructura" de acuerdo a la proposición del estructural funcionalismo; aunque, a pesar de su difusión e importancia en la ciencia política norteamericana, no parece ser la más adecuada y fecunda. Desde un punto de vista lógico y epistemológico, por el contrario, resulta más correcto y fructífero establecer la relación dialéctica entre "funcionalismoorganicismo". Más que a una "estructura funcionando" la categoría de "función” se liga coherentemente a una concepción "orgánica” (Bergeron, $1965)^{6}$. El Estado puede ser considerado por niveles y a través de las diversas funciones que cumple. Por la naturaleza del Estado las funciones son siempre de control.

Es decir, para Bergeron (1993) la noción de “función” está asociada a la de "control". Ambas son herramientas que permiten muchas sutilezas en el análisis y que están relacionadas con otros instrumentos que constituyen parte importante del bagaje instrumental que se requiere en el análisis para abordar su objeto de estudio.

Este enfoque considera al Estado como una totalidad orgánica funcional de control en la que se distinguen distintos niveles: en primer término: el Régimen donde se dan las funciones de "legitimación” y "seguridad”; es el nivel del Estado “controlador” (Bergeron, 1993, p. 85-102). En segundo, en el gobierno se manifiestan las funciones actuantes de conducción del poder en el plano de la "ejecución”; es el nivel del Estado "controlando” (Bergeron, 1993, p. 35-60). Y en tercer lugar la sociedad, con las funciones de participación, es el nivel del Estado "controlado" (Bergeron, 1993, p. 61-84).

Estos tres niveles pueden ser analizados de abajo hacia arriba y de arriba hacia abajo, en el primer caso, se refiere a la lucha política por participar en la en la determinación de las autoridades e instituciones que deberán hacer cumplir obligatoriamente las normas con el fin que aseguren las distintas expresiones de valores de diversos sectores que aspiran a que les sean reconocidos y respetados. Desde "arriba hacia abajo" hace referencia a las políticas públicas que el "gobierno" impone a la ciudadanía; que en el caso del ejercicio del poder significa hacer cumplir las reglas de la participación y su legitimidad.

Visto de otro modo, el régimen (arriba hacia abajo) determina las condiciones del ejercicio del poder, tanto del gobierno, como de la sociedad, al mismo tiempo que otorgan la soberanía en su actuación. El gobierno siendo legitimado por el régimen puede actuar para modificar el statu quo de la sociedad en las situaciones que juzgue como problemáticas. La sociedad, al igual que el gobierno es legitimado por el régimen y habilitado para participar de los asuntos públicos y hacer demandas al gobierno para atender los problemas públicos, quién debe cumplirlas.

Sin embargo, varias son las tareas que Bergeron deja pendientes. En efecto, el término de "función" propuesto por el investigador canadiense 
es prioritariamente un conjunto de “acciones humanas” políticas; que puede ser considerada, a su vez, como “trabajo político”. Sustituir la noción de función de trabajo, podría agregar a la "función” su relación con la materia o naturaleza fáctica que Bergeron no considera suficientemente. Por ello, la propuesta es sustituir los distintos "niveles funcionales” que expresan la acción del Estado por las “políticas” e "instituciones” para dar concreción a la funcionalidad general, en un momento determinado, del Estado.

Las políticas y las instituciones como expresiones efectivas de la funcionalidad estatal concurren al mismo tiempo y constantemente para mantener viva la totalidad estatal específica. En el plano de las políticas públicas no se trata de una función en general: sea de legitimidad o de administración de justicia, por ejemplo, sino de una determinada política que se expresa en leyes obligatorias determinadas para hombres y no para dioses (Bolívar Espinoza \& Cuellar Saavedra, 2008).

A la teoría funcional de Bergeron se integra el esquema las políticas efectivas y las instituciones específicas que ponen en movimiento el sistema orgánico. Esto quiere decir que cuando se habla de niveles funcionales o de funciones en cualquier sentido en que se utilice la categoría de "función” debe pensarse al mismo tiempo en órganos ("instituciones vivientes”) y en políticas (ideas en acción).

Por otra parte, la transformación de "función” “a “políticas” por medio de la noción de "trabajo” se hace más evidente: las políticas harían referencia a cumplir no sólo funciones sino "trabajos” de control. Sin embargo, el nivel funcional en el que se mueve Bergeron, aparece, por el momento, más que suficiente para la propuesta del análisis del Estado desde el análisis de las políticas públicas como estrategia metodológica para eliminar el haló filosófico y el carácter general que tiene el Estado.

\section{Estado y políticas públicas}

\section{Las políticas públicas como indicadores de la acción del Estado}

Si bien el análisis de los países requiere de la concepción del Estado orgánico y funcional, como soporte heurístico no es menos cierto que esta idea es secundaria en el proceso analítico; en este aspecto, lo primero es la acción o el funcionamiento del Estado expresado en políticas. Pero, así como hay distintos niveles de funciones también hay niveles en las políticas: de seguridad y legitimidad desde y para el régimen; de gobierno y legislación, y de administración y de juzgar desde y para el gobierno; de participación desde y para la sociedad. Las políticas, en este sentido se entienden como acciones ligadas por una idea, o una función (Bolívar Espinoza A. , 1997a; Bolívar Espinoza \& Flores Vega, 2014). Las políticas son estatales y también son públicas; incluso, muchas políticas privadas son también públicas (Bozeman \& Coord, 1998), es decir, todas son diseñadas y ejecutadas por o con la participación significativa del gobierno, con 
menor o mayor participación de la sociedad y legitimadas por el régimen (la Constitución).

Todas son funciones y políticas, pero no todas tienen el mismo valor heurístico en el análisis. Cuando se quiere caracterizar y establecer el cambio en un Estado determinado una opción útil es elegir como enfoque las políticas públicas en dos sentidos: en forma puntual, mediante el análisis en detalle de una o varias políticas que se consideran significativa; o, en conjunto, a través del estudio del impacto y composición que tienen ciertas políticas en el funcionamiento global del Estado. Ambas perspectivas son complementarias. La primera supone una visión secuencial que va desde el problema a la evaluación de los resultados de la política propuesta para resolverlo. La segunda se expresa en el estudio de la puesta en marcha políticas vistas en sus mutuas dependencias; la expresión más comprensiva de estas políticas se encuentra en las políticas constitucionales o de reforma constitucional (Bolívar Espinoza \& Flores Vega, 2014).

El “enfoque de políticas” ha permitido analizar las acciones del Estado como una especialidad. Es una alternativa viable, ante la limitación de las ciencias sociales, para analizar la acción pública vista tanto en su conjunto como en sus particularidades. El "análisis de políticas” trata de considerar las políticas gubernamentales, interpretándolas, tanto en sus efectos como en sus respuestas a la sociedad. Intenta determinar los criterios de selección y exclusión de los problemas públicos a resolver por el gobierno. Las razones que determinan la elección de los instrumentos de los cursos de acción. Los criterios y los énfasis de la implementación y, los valores que juegan en la selección y evaluación. Las causas que originan la inercia en las políticas su corrección o reemplazo ${ }^{7}$.

Mucho más que una ciencia deductiva el análisis de políticas se caracteriza por el arte de hacer preguntas. Se trata de verificar la pertinencia de las dudas confrontándolas con los hechos y con una explicación. Enfoca diversos campos y ofrecer una alternativa metodológica para abordarlos. En esta medida permite, desde una perspectiva acotada, tratar de la aparición y naturaleza del Estado, de la esencia de la política, y del impacto de la acción pública en la sociedad.

Mediante una pluralidad de métodos que descomponen analíticamente la acción pública, la compara diversas situaciones y con distintos grados de profundidad. Supone el análisis del contenido y proceso de las políticas. Analiza sectores, tipos de autoridades políticas y determina épocas. Estas características hacen posible una gran capacidad heurística y operacional. La ciencia de políticas representa un avance considerable en el estudio como en la elaboración de la acción pública, específica y actual. Pese a las divergencias, las variantes nacionales, la pluralidad teórica y la diversidad metodológica, constituye un aporte consolidado (Meny \& Thoenig, 1992). 


\section{¿Cómo analizar los estados desde las políticas públicas?}

En el análisis (AFPICP) una de las instancias privilegiadas, aunque no la única, para determinar los indicadores que permitan determinar la especificidad y contenido global de la transformación de Estado, lo constituye la Constitución Política del Estado y las instituciones que la apoyan. Varias son las razones por las cuales esta perspectiva de análisis puede ayudar a resolver con ventaja los problemas derivados del análisis de cambio del Estado o del régimen político en países de institucionalización reciente y diferida, y, en especial, los que vive México desde principios de la década de los ochenta. En primer término, el tratamiento del Estado desde el punto de vista de su “acción” (Muller \& Surel, 1998; Muller, 2002).

En segundo la opción empírica que supone la preeminencia de los hechos. En tercero, la consideración prioritaria del análisis multidisciplinario y de los estudios comparativos. En cuarto lugar, su carácter pragmático. Todas estas razones encuadran y supeditan las alternativas teóricas y metodológicas generales y abstractas al universo de los problemas y soluciones específicas de la acción pública.

Las perspectivas ideológica, jurídica y, por tanto, también la política de la Constitución muestran, el grado y la forma como se expresa el orden político; sea en disposiciones discursivas, leyes o costumbres, formalizadas o no, que afirman una situación global o específica de la vida social y, de su destino viable. Desde el punto de vista del análisis de políticas públicas interesa el Estado en funcionamiento, es decir, las políticas públicas. Este tipo de políticas, en primer término, son acciones del gobierno, es decir, políticas públicas; en segundo, que están destinadas al funcionamiento, la creación o disolución de instituciones; en tercero, porque a pesar de ser "políticas públicas”, están relacionadas íntimamente con lo político y la política.

El análisis de los estados desde las políticas públicas sortea la antítesis rígida prevaleciente en algunos procedimientos en el seguimiento de procesos políticos y en el conocimiento de la estructura estatal. Por una parte, se aleja de la reflexión deductiva de esquemas teóricos generales del Estado. Por otra, desconfía de los estudios empíricos y particularistas. Sus postulados no se derivan de una perspectiva metodológica abstracta, a priori. Utiliza todos los recursos metodológicos como herramientas en función de la comprensión del “orden”, definido operacionalmente. Es utilitarista y pragmático. Procede en función de la capacidad explicativa mayor de la concepción y técnica adoptada. Tiene la pretensión de analizar el presente político en forma sistemática y rigurosa, suponiendo que la base para la comprensión del orden político se encuentra en los datos observables. Parte de los fenómenos e intenta ordenarlos mediante un sentido explicado por la misma realidad empírica, aunque evidentemente, no exclusivamente. La perspectiva positivista y empirista participa como instancia necesaria y asimilable con la posición dialéctica. Finalmente, distingue también entre los procesos reales concebidos ontológicamente que son incomprensibles 
y la percepción ordenada y provisoria que se construye a partir de la experiencia sensible.

Al considerar las políticas gubernamentales, desde el punto de vista del "Estado en acción" no sólo se recupera su carácter técnico, sino también los condicionantes y efectos de la dimensión social. Gracias a la consideración de las políticas públicas en una concepción integrada se vuelve posible considerar el cambio desde una perspectiva global. En definitiva, la evolución teórica en las ciencias del derecho y de las políticas públicas, junto con las transformaciones políticas de las últimas décadas ha hecho posible la idea de estudiar los preceptos constitucionales que limitan el poder y, sobre todo, las instituciones que lo controlan. Este acontecimiento es aún más significativo en los países de institucionalidad reciente, y, en especial, en América Latina.

La construcción de estrategias para el análisis del Estado desde las políticas públicas y su cambio en países periféricos, en especial en América latina, viene de larga data y ha sido abundante aunque difícil e incierta; comienza con fuerza a principios del siglo XIX acompañando los procesos de independencia y todavía está pendiente en los intentos por explicar la reforma del Estado; en este proceso de construcción teórica y metodológica no han estado ajenos los pensadores franceses ${ }^{8}$. Por otra parte, si bien los paradigmas clásicos sobre el Estado han sido fundamentales para desarrollar algunos campos de investigación no han sido suficientes y muchas veces han resultado contraproducentes para la comprensión de las nuevas realidades sociales emergentes ${ }^{9}$;

Dos son los problemas fundamentales, entre muchos, enfrenta el análisis de políticas públicas en América latina: la concepción del Estado y de la sociedad civil; $y$, el proceso internacional en el que se desarrolla. El primero, aparece: por una parte, como producto problemático de la suma de las dificultades internas de las estrategias analíticas criollas más las disputas clásicas europeas, especialmente, las derivadas de la alternativa pluralista; por otra, el complejo avance que ha supuesto la "creación” de nuevas categorías a los procesos históricos emergentes ${ }^{10}$. El segundo problema, se revela en el doble proceso que supone: las exigencias del desarrollo de las fuerzas productivas y de las relaciones internacionales al intervenir en los procesos internos de legitimación y seguridad; y, a la inversa, el análisis de la "reproducción ampliada" a escala nacional que conlleva en forma inherente, las relaciones de producción a escala internacional.

Son varias los calificativos que ha recibido el Estado en América latina de acuerdo a las diversas estrategias de análisis; el término de "Estado periférico" incluye a la mayoría posturas teóricas en la medida que destaca un rasgo general compartido por todas: la de encontrarse ligados pero al margen de los países centrales. Los países periféricos constituyen de hecho, a esta altura del desarrollo de las fuerzas productivas internacionales, en su gran mayoría, “objetos orgánicos” susceptibles de ser considerados como identidades. 
El “Estado”, el “sistema político”, o la “formación social” específica nacional, deben ser considerados como límites especiales inmediatos, cuya necesidad y permanencia está requerida por el desarrollo del capitalismo a escala internacional; sin embargo, esto no excluye y al contrario exige, que el punto de partida y de llegada sea el análisis inmediato de las situaciones específicas de dominación. Estos fenómenos deben ser vistos como respuestas a desafíos internacionales y a contradicciones específicas de los proceso políticos internos de los "países periféricos” Cualquier intento de subordinación de "lo periférico” en lo “desarrollado”, ya sea en el sentido de deducción o comprensión parcialmente constituye ideología en su sentido más pobre.

El tratamiento del desarrollo “periférico” supone considerar el desarrollo específico en relación con las determinaciones de la reproducción del capital a escala internacional donde el Estado nacional aparece como primer contexto del desarrollo interno. Así, el peronismo, no es exclusivamente, una forma de social democracia mezclada con populismo militarista, ni el "pinochetismo" una forma subdesarrollada de fascismo, ni el priísmo una “dictadura perfecta” etc., tampoco es suficiente la caracterización de realidades folclóricas.

\section{El tema del Estado en América Latina como institucionalización reciente}

En América latina y en el resto del tercer mundo se ha ido acuñando el concepto de "reforma del Estado" para explicar y designar este proceso de cambio de las últimas décadas. El término, sin embargo, no deja de manifestar vastas imprecisiones en el terreno académico y en la contienda política. Generalmente se le ha vinculado exclusivamente al dominio económico nacional e internacional. Otras veces se le ha asimilado a la necesidad de establecer nuevas reglas de funcionamiento político que asegure la democracia.

Algunas expresiones teóricas han hecho la síntesis de ambas posiciones precisando su carácter como "tránsito hacia la democracia” que exige la liberalización del mercado. Tanto la reforma del Estado vista desde el mercado como el perfeccionamiento de la democracia constituyen uno de los grandes desafíos para la política y las ciencias sociales modernas. En el caso del análisis la reforma del Estado se entiende como cambio en el ejercicio del poder en el régimen y como cambio hacia otro sistema político independientemente que este deba llevar implícitos necesariamente, la preeminencia del mercado en la construcción de la democracia.

La elección del gobierno o del régimen político como punto de vista para enfrentar los problemas de transformación del Estado se justifica sobre todo en países en vías de desarrollo. En primer lugar, por la fácil disposición de materiales documentales para su análisis y, por constituir un uni- 
verso relativamente restringido definido formalmente por disposiciones jurídicas. Si bien no le confieren límites absolutos acotan el entorno de su influencia y determinan la legalidad y legitimidad de las acciones públicas y privadas. En segundo lugar, y como una argumentación, de más peso y contenido, las políticas públicas son monopolio de la acción del gobierno $^{11}$. Por último, y en tercer lugar, no parece aventurado afirmar y comprobar que las acciones de la sociedad civil y de la sociedad política en México, pueden ser vistas desde la perspectiva gubernamental sin que pierdan su significación original. Por lo demás, en las no muy frecuentes ocasiones que estas acciones aparecen en abierta independencia y aún beligerancia con la autoridad gubernamental, pronto son incluidas en un esquema en que la hegemonía del aparato se impone.

La propuesta aquí presentada considera al Estado en funcionamiento en América latina como periférico y de "institucionalización reciente y diferida”, es decir, un Estado donde la encarnación de las instituciones “clásicas” y la implementación de las políticas de control en la sociedad es muy reciente y difiere del carácter originario de las centrales; encontrándose en proceso la conformación de una institucionalidad acorde con las exigencias de la sociedad.

Los regímenes periféricos son producto de "injertos" y "rechazos" de modelos clásicos en situaciones específicas e irrepetibles; constituyen nuevas normatividades del ejercicio del poder donde las constituciones y las instituciones mezclan formas autóctonas, con resabios de estructuras coloniales, y superestructuras centrales. En general, son países que habiendo sido colonizados, ocupados o sujetos a dictaduras, en la etapa de independencia o en vías de obtenerla, han "importado" una o varias "formas" de "Estados constitucionales". En estas circunstancias, sólo después de varios años de pasada la independencia a principios del siglo XIX en América Latina, y a mediados del XX en África y Asia, comienzan a imponerse de hecho y con lentitud, las instituciones trasplantadas, debido a estas circunstancias:

• está pendiente la construcción de instituciones inherentes al desarrollo del Estado constitucional, para su buen funcionamiento;

• sólo algunas instituciones cumplen las funciones para las cuales fueron creadas;

- la mayoría de las veces estas funciones se realizan de forma y con contenidos diferentes;

- muchas instancias de sociabilidad autóctonas permanecen;

- y, por último, también se crean nuevas instituciones que, conservando su contenido de origen, se adaptan a las condiciones específicas donde operan, incorporando formas institucionales antiguas de la localidad. 
El calificativo de "reciente" se refiere a la asimilación efectiva de modelos institucionales externos, no a la fecha en que fueron incorporados formalmente. La mayor parte de los países del mundo han pasado por esta forma de institucionalización. Sin embargo, debido a que las formas impuestas desde el exterior son formas cercanas a la tradición local, algunos países se adaptan rápidamente a las nuevas normas y encuentran rápidamente su propia Constitución; otros, han "diferido" hacia nuevas formas de normatividad en la que las crisis son permanentes. La intensidad y forma de la adaptación y los "rechazos" o el grado de desviación de modelos “injertados" define cada uno de los regímenes particulares en forma irrepetible. El hecho que estos países no posean normas que correspondan a las formas efectivas de ejercer el poder produce una inestabilidad permanente. Este proceso sucede hasta encontrar una adecuación mayor en los valores de la sociedad con los principios generales del Estado y la normatividad del gobierno; o, por el contrario, estas sociedades entrarán en procesos autoritarios hasta generar políticas constitucionales fundadas en reales pactos nacionales.

La construcción, desarrollo y formalización de las políticas públicas en los países periféricos han sido diferentes a las de los países maduros institucionalmente a consecuencia que el Estado en el caso de los primeros se modela al mismo tiempo que la sociedad; en estas sociedades las políticas públicas han debido ser muy abundantes y todavía enlazan aspectos institucionales formales clásicos con instancias políticas en desarrollo y, formas inéditas de ejercicio del poder surgidas de exigencias propias. El proceso de "implante" y de "rechazo" de las instituciones paradigmáticas configurará la especificidad de las sociedades emergentes (Meny \& Thoenig, 1992). Este fenómeno produce efectos y relaciones totalmente novedosas en la forma como las instituciones paradigmáticas de los países desarrollados se comportan en nuevos medios.

Por ello, la reforma del Estado constituiría también un proceso único en cada país; que deberá contar con indicadores que puedan mostrar los avances o retrocesos en este proceso de cambio; estos referentes se construyen a partir de las políticas públicas o gubernamentales en especial de reforma constitucional que definen a su vez políticas (Lechner, 1988)

\section{Conclusión}

La reciente preocupación por el Estado y sus instituciones ha generado nuevos problemas aunque, al mismo tiempo, ha exigido nuevas soluciones. Las críticas al análisis sistémico a la elección racional hasta no hace mucho, hegemónicos en el análisis de políticas, han exigido nuevas perspectivas metodológicas y nuevos tratamientos teóricos sobre el Estado. Los intentos de ligar la reflexión sobre la política y las políticas han permitido, entre otros, desarrollos interesantes en el diseño intelectual de las políticas y en el entorno que las rodea. 
La propuesta aquí planteada se puede resumir en: la "acción del Estado" sólo se puede dar a través de "políticas públicas”; y, estas necesariamente deben ser gubernamentales. Tanto las políticas públicas de origen privado como las internacionales, también, deben vincularse necesariamente a las políticas gubernamentales. No es imposible imaginar que haya políticas predominantemente gubernamentales, en su origen, que adquieran el carácter público. Muchas de ellas jalonan los procesos democratizadores en todos los países de institucionalización reciente. Cuando las políticas gubernamentales se centran en las reformas constitucionales o abrogan la Constitución quiere decir que el cambio en la naturaleza del ejercicio del poder es inminente.

Los procesos de cambio o permanencia "cercanos", de nuestra región, requieren de nuestra inmediata preocupación para nuestra formación a fin de poder estar mejor preparados para abordar procesos más lejanos: los cambios de régimen, el comportamiento de las instituciones, la reacción de las sociedades frente al poder, la búsqueda del orden o la ruptura del mismo, los mecanismos de participación y la comprensión de la violencia requieren de la proximidad empírica, de un lenguaje apropiado y de un conocimiento tácito elevado. Así lo hicieron todos los grandes clásicos del pasado como los contemporáneos. Las ideas diagnósticos, enfoques, y experiencias analíticas tienen siempre que estarse poniendo a prueba con la realidad efectiva inmediata.

América Latina ha pasado estos últimos decenios por procesos significativos de cambio, no solo en las estructuras institucionales constitutivas si no en la vida social cotidiana los que difícilmente pueden ser analizadas a través de categorías o modelos vacíos a priori. No se trata de la necesidad de un enfoque chovinista ni de construir modelos teóricos y metodológicos propios, a nuestra nación o continente, se trata, especialmente en los procesos de aprendizaje e investigación universitarios -que no es poco decir- de abordar los procesos sociales vividos y perceptibles en medio de una problemática que nos desafía constantemente y requiere de una comprensión mediana antes de volar a alturas y distancias mayores. 


\section{Notas}

${ }^{1}$ En la región fue recurrente el uso de "reforma del Estado", paradójicamente el tema se planteó como la necesidad de la reforma del Estado pero sin saber qué era Estado, ni en qué plano se consideraba; y, sin embargo, aparecía como lugar común la reforma de “algo” que evocaba múltiples y controvertidas significaciones (Bolívar Espinoza, 1997).

${ }^{2}$ Autores como Muller (2002, págs. 59-62) han identificado una serie de límites al enfoque secuencial, tanto con el orden de las etapas, como con las etapas mismas.

${ }^{3}$ A pesar de la presencia indiscutible del Estado, la ambigüedad y las dificultades para materializar al Estado como un campo de estudio, permitió que autores como Gérard Bergeron (1993), quién será uno de los defensores y responsables de traer de nueva cuenta al Estado al centro de la ciencia política, se refirieran al Estado con ambigüedad.

${ }^{4}$ Si bien podemos señalar la perspectiva elitista de Mosca y Pareto o el corporativista de Schmitter, estos pueden ser entendidas como casos particulares del enfoque pluralista.

${ }^{5}$ Gérard Bergeron (1965) se extiende ampliamente para distanciar su concepción “orgánica-funcional” de la "estructural-funcionalismo” (pp. 95-104).

${ }^{6}$ Lo que se enfatiza con la idea de un Estado orgánico es que los conglomerados humanos, viven en constante movimiento de independencia y auto reproducción; y que funcionan de diferentes maneras pues, en su cambio, están consideradas la conciencia y la conducta, pública y privada. Pero, que a su vez, las "acciones humanas" vistas en totalidad y en transformación, están influenciados por un medio que las afecta el que también es creado y recreado constantemente por ellas (Bolívar Espinoza A. , 1997a).

${ }^{7}$ El análisis de políticas trata de “(...) buscar saber cómo y por qué determinados problemas son calificados de públicos y a otros se les niega tal calidad cómo y por qué el gobierno incluye ciertos problemas en su agenda y excluye otros; cómo y por qué los percibe de una cierta manera y deja de lado otras definiciones plausibles, tal vez más relevantes; cómo y por qué los gobiernos tienden a elegir ciertos cursos de acción, a privilegiar ciertos instrumentos, a acentuar ciertos aspectos de la acción pública, y descartan otros cursos, instrumentos, operadores, aspectos; cómo y porqué se eligen ciertos patrones de implementación y se prefieren ciertos criterios valorativos en la elección y evaluación de las políticas; cómo y por qué se decide continuar con las viejas políticas, tal vez corrigiéndolas o se decide cancelarlas o darlas por terminado” (Aguilar, 1992: t. 1 p. 52).

${ }^{8}$ Entre los muchos trabajos desarrollados en Francia respecto de diversos aspectos del Estado latino americano, destacan: los de Lambert (1963 y 1987) que muestran la precariedad del equilibrio de poderes, la preponderancia del presidencialismo; los propiciados por Georges Burdeau en su colección "Comment ils sont gouvenrnés” donde destacan los trabajos sobre América latina y México de Tadeusz Wyrwa que dan cuenta del "exotismo politique du monde hispanique" (1972:p. 1); los aportes al estudio de los gobiernos militares de de Alain Rouquié (1982, 1987, 1992) y, más recientemente, Couffignal (1992), Olivier Dabène (1997), Guy Hermet (1996), algunos de los cuales ofrecen amplias bibliografías en los que aparecen los aportes de ese país. 
${ }^{9}$ Después de los abundantes aportes de datos de los cronistas coloniales y criollos demasiado localistas; de la construcción de identidades nacionales de los grandes historiadores del siglo XIX muy sesgados de ideología chauvinista; del aporte teórico del marxismo que pecó de generalidad e ideología; de las nuevas técnicas estadounidenses de los años cincuenta marcadas por las limitaciones pluralistas; de los análisis desde la periferia elaborados en la CEPAL y la "teoría de la dependencia” demasiado urgidas por el desarrollismo; las perspectivas analíticas para el estudio de la reforma del Estado si bien se encuentran mejores equipadas, no es menos cierto que no han producido una noción operacional del Estado que haga posible el análisis.

${ }^{10}$ La Teoría de la dependencia aportó algunas categorías que contribuyeron a determinar la especificidad de las "relaciones sociales" que están a la base de una esfera pública propia, entre otras. Todavía hoy, se utilizan corrientemente el "clientelismo” y el “corporativismo” para explicar deductivamente sistemas complejos.

${ }^{11}$ Es necesario, sin embargo, establecer en la reflexión sobre las instituciones, las políticas en general y, especialmente las políticas de reforma constitucional el papel esencialmente gubernamental que poseen estos fenómenos cuando se aplican a países desarrollo diferido. 


\section{Bibliografía}

Andara M., A. E. (2007). La segunda generación de reforma del Estado y su efecto en la administración pública local de América Latina. Revista Provincia, No. 17, 77-105.

Bergeron, G. (1965). Fonctionnement de L'État. Paris, Francia: Librairie Armand Colin.

Bergeron, G. (1993). L'État en fonctionnement. Paris, Francia: L’Harmattan.

Bolívar, A. (1997a). Un instrumento de análisis de la reforma del Estado. Las políticas institucionales de Control: el caso de México (19821997) (Tesis Doctoral, Versión en Español). Universidad de Paris I, Sorbona, Paris, Francia.

Bolívar, G. A., \& Flores Vega, L. (2014). Un programa de investigación sobre la reforma del estado en América Latina. Análisis funcional de políticas institucionales de control político. México, D.F, México: Eon.

Bolívar Espinoza, G., \& Cuellar Saavedra, Ó. (2008). La república legítima y el orden político en Rousseau: principios de composición e imágen del Estado en equilibrio. Polis, Revista Latinoamericama 7(20) http:/ /dx.doi.org/10.4067/S0718-65682008000100013

Bozeman, B., \& Coord). (1998). La gestión pública su situación actual. México D.F, México: FCE.

Evans, P. (2001). El Estado como problema y como solución. En A. Camou, Los desafíos de la gobernabilidad (págs. 131-176). México D.F, México: FLACSO-IISUNAM-Plaza y Valdés.

Filgueira, F. (2009). El desarrollo maniatado en América Latina. Estados superficiales, desigualdades profundas. Buenos Aires, Argentina: CLACSO-CROP.

Halperin Donghi, T. (2013). Historia contemporánea de América Latina. Madrid, España: Alianza.

Jobert, B. (1994). El Estado en acción; la contribución de la políticas públicas. Revista Ciencia Política (36). págs. 79-104. Recuperado de http:/ /www.icpcolombia.org/dev/wp-content/uploads/1994/07/ el_estado_accion_contribucion_politicas_publicas.pdf

Jobert, B., \& Muller, P. (1987). L'État en action. Politiques publiques et corporativisme. París, Francia: PUF.

Lechner, N. (1988). Los patios interiores de la democracia. Subjetividad y política. Santiago, Chile: FLACSO. 
Meny, Y., \& Thoenig, J.-C. (1992). Las políticas públicas. Madrid, España: Ariel.

Muller, P. (2002). Las políticas públicas. Bogotá, Colombia: Universidad Externado de Colombia.

Muller, P., \& Surel, Y. (1998). L’analyse des politiques publiques. Paris, Francia: Montchrestien, clefs/politique.

North, D. (1995). Instituciones, cambio institucional y desempeño económico. México D.F, México: FCE.

O’Donnell, G. (2004). Acerca del estado en América Latina contemporánea: diez tesis para discusión. En La democracia en América Latina. Hacia una democracia de ciudadanas y ciudadanos: contribuciones para el debate (págs. 149-191). Buenos Aires, Argentina: Programa de Naciones Unidas para el Desarrollo.

Oszlak, O., \& O’Donnell, G. (1976). Estado y políticas estatales en América latina: hacia una estrategia de investigación. Documento CEDES/ G. E. FLACSO/ núm. 4. Santiago, Chile: FLACSO.

Roth Deubel, A.-N. (2006). Políticas públicas. Formulación, implementación y evaluación. Bogotá Colombia: Aurora.

Skocpol, T. (2007). El Estado regresa al primer plano: Estrategias de análisis en la investigación actual. En Lecturas sobre el Estado y las políticas públicas: retomando el debate de ayer para fortalecer el actual (págs. 169-202). Buenos Aires, Argentina: Jefatura de Gabinete de Ministros .

Wolfe, A. (1980). Los límites de la legitimidad, contradicciones políticas del capitalismo contemporáneo. México D.F, México: Siglo XXI.

Yocelevzky Retamal, R., \& Cuéllar Saavedra, Ó. (2011). El misterio latinoamericano. Polis, Revista de la 10(29), 209-224. https://dx.doi.org/ 10.4067/S0718-65682011000200010

Recibido: 15.09.17

Aceptado: 03.11.17 